'Departamento de Dermatología, Facultad de Medicina, Universidad de Chile. Santiago, Chile.

2Instituto de Nutrición y Tecnología de los Alimentos (INTA), Universidad de Chile. Santiago, Chile.

aResidente Programa de Especialización en Dermatología. Escuela de Postgrado, Facultad de Medicina, Universidad de Chile. bPhD.

Los autores declaran no tener conflictos de interés. Trabajo no recibió financiamiento.

Recibido el 28 de marzo de 2020, aceptado el 16 de junio de 2021 .

Correspondencia a: Dra. Magdalena Araya INTA, Universidad de Chile. El Líbano 5524. Santiago, Chile. maraya@inta.uchile.cl

\section{Dermatitis herpetiforme y enfermedad celíaca. Del intestino a la piel}

\author{
CAROLINA GARCÍA ${ }^{1,2, a}$, MAGDALENA ARAYA,b
}

\section{Dermatitis herpetiformis and celiac disease}

Dermatitis herpetiformis is an autoimmune chronic blistering disease, considered a skin manifestation of celiac disease. Being both conditions multifactorial, they share some genetic traits and pathogenic mechanisms, which are responsible for the typical skin and gastrointestinal manifestations. In dermatitis herpetiformis, skin and other lesions heal after gluten-free diet and reappear shortly after its reintroduction to complete diet. Prevalence of celiac disease is 1\% in the population, and approximately $13 \%$ of patients with the disease develop dermatitis herpetiformis. Diagnosis of celiac disease has progressively increased in recent decades, while clinical manifestations become more and more diverse. Given the current high frequency of skin lesions in celiac patients, in this review we update relevant aspects of the epidemiology, pathogenesis, clinical presentations, treatment and follow up of dermatitis herpetiformis, as a contribution to improve the management of both conditions.

(Rev Med Chile 2021; 149: 1330-1338)

Key words: Celiac Disease; Dermatitis Herpetiformis; Transglutaminases.
L a dermatitis herpetiforme (DH) es una enfermedad ampollar crónica autoinmune de base genética, considerada una manifestación cutánea de la enfermedad celíaca (EC). Ambas condiciones comparten aspectos genéticos y etiopatogénicos, que conducen a alteraciones cutáneas e intestinales que mejoran con una dieta libre de gluten (DLG). En nuestro país, la frecuencia de EC es cercana al 1\% de la población, comparable a la reportada en otros países ${ }^{1-3}$. Se estima que alrededor de 13\% de los pacientes con EC desarrollan $\mathrm{DH}^{4,5}$. Los estudios epidemiológicos sobre $\mathrm{DH}$ son escasos y en su mayoría noreuropeos, donde este trastorno es más común. Dado el aumento de diagnósticos de EC, la amplia diversidad de sus manifestaciones clínicas y la alta frecuencia de lesiones cutáneas en nuestros pacientes (datos no publicados), hemos considerado de interés hacer una revisión narrativa que ponga al día ambas condiciones.

\section{Material y Métodos}

La revisión utilizó bases de datos MEDLINE y PubMed Central (PMC) (acceso a textos completos), usando las palabras "celiac", coeliac", "dermatitis herpetiformis", en artículos publicados entre 1900-2019, en inglés o español, cualquier edad y sexo. La literatura pertinente es escasa, por lo que se revisaron, además, las referencias que aparecían en artículos índices.

\section{Resultados}

Duhring (1884) describió la DH cuatro años antes que Samuel Gee publicara su trabajo en EC. La enteropatía fue descrita en $1966^{6}$ y en 1997 se descubrió la inmunogenética compartida entre DH y EC, asociada a HLA-DQ $2^{7}$. Luego se describió la transglutaminasa tisular 2 (tTG2) como el autoantígeno contra el cual se detectan anticuerpos 
en pacientes celíacos ${ }^{8} \mathrm{y}$, posteriormente, también se demostró su presencia en $\mathrm{DH}^{9}$. En 2002, Sárdy identificó la transglutaminasa epidérmica (tTG3) como autoantígeno en la $\mathrm{DH}^{10}$. Así, se demostró que los autoantígenos principales son distintos en ambas enfermedades, pero comparten ciertos epítopes comunes, que en ocasiones permiten la reacción cruzada.

\section{Epidemiología}

$\mathrm{La} \mathrm{DH}$ es poco frecuente, su prevalencia varía según la raza y país estudiado $(75,3 / 100.000$ en Finlandia ${ }^{4}, 30 / 100.000$ en Reino Unido ${ }^{5}$ ). En afroamericanos hay reportes puntuales y es infrecuente en poblaciones asiáticas ${ }^{11}$. En Japón se ha planteado que la asociación entre DH y EC sería más débil que en países occidentales, ya que los pacientes con $\mathrm{DH}$ japoneses carecen de haplotipos HLA DQ2/DQ8, de compromiso intestinal y de los marcadores serológicos típicos de $\mathrm{EC}^{12}$.

En contraste con la EC, la incidencia de la DH ha disminuido significativamente en las últimas décadas. Una hipótesis actual postula que los avances en la capacidad diagnóstica de EC hacen que su reconocimiento y tratamiento sean tempranos, no dando tiempo para el desarrollo de $\mathrm{DH}^{4,5}$, que se considera consecuencia de la EC no tratada ${ }^{10}$. $\mathrm{La} \mathrm{DH}$, generalmente, se diagnostica durante la adultez ${ }^{4,5} \mathrm{y}$ su mayor frecuencia ocurre entre los 50 y 69 años. También, la mayoría de los estudios poblacionales describen que $\mathrm{DH}$ predominaría en hombres (hasta 2:111). Sin embargo, estudios más recientes muestran que la proporción entre hombres y mujeres puede ser cercana a $1: 1^{4,5}$.

\section{Etiopatogenia}

La fisiopatología de DH y EC implica una interacción compleja entre factores genéticos, inmunes y ambientales. $\mathrm{Al}$ igual que en EC, existe una fuerte asociación con HLA-DQ2, portados por el $86 \%$ de los pacientes DH (versus $25 \%$ en controles), y en menor medida con HLA-DQ8 ${ }^{7}$. En EC, las descripciones europeas son semejantes ${ }^{13}$. En Chile, la frecuencia y distribución de estos heterodímeros en EC es distinta, siendo comparativamente menor HLA-DQ2, mayor HLA-DQ8, con una frecuencia de HLA-DQ7-que en Europa no se menciona- semejante a la de HLA-DQ8 ${ }^{14}$. Se desconoce si estas diferencias ocurren también en pacientes $\mathrm{DH}$.

Se postula que DH se inicia con el daño de la mucosa intestinal en el contexto de una EC oculta, gatillada por la ingesta de gluten en individuos genéticamente susceptibles. La aplicación tópica o intradérmica de gluten no desencadena lesiones típicas de $\mathrm{DH}^{15}$. En la mucosa intestinal, la gliadina actúa como sustrato para la $\mathrm{tTG}^{8}$, con la que forma complejos covalentes (péptidos deamidados de gliadina o PDG) que tienen alta afinidad por HLA- DQ2/DQ8 en las células presentadoras de antígenos, lo que resulta en una respuesta inmune contra el complejo tTG2/PDG. Los linfocitos T $\mathrm{CD} 4+$ activados inician la respuesta mediada por citoquinas Th1, que daña la mucosa ${ }^{16}$ (Figura 1). La respuesta Th2 da como resultado la formación de anticuerpos IgA contra la gliadina y/o el complejo tTG2/PDG y/o el endomisio, siendo la tTG2 el autoantígeno diana ${ }^{8}$ (Figura 1). En DH, la tTG3 es el autoantígeno principal ${ }^{10}$. Entre tTG2 y tTG3 la conservación aminoacídica es 38\%, con hasta $64 \%$ de homología en ciertas regiones ${ }^{17}$. Después de una exposición prolongada a gliadina, se desarrollan anticuerpos circulantes contra epítopes comunes a ambas transglutaminasas, incluidos anticuerpos IgA que tienen avidez baja por tTG2 y muy alta por $\mathrm{tTG} 3^{10}$. Datos recientes respaldan que, en $\mathrm{DH}$, la producción de autoanticuerpos IgA anti-tTG3 ocurre en el intestino delgado ${ }^{17}$. Se ha sugerido que los complejos inmunes circulantes IgA-tTG3 luego se depositan en las papilas dérmicas, formando los depósitos característicos de la enfermedad ${ }^{10}$ (Figura 2). En la piel, el depósito de IgA-tTG3 activa al complemento y provoca infiltración de las papilas por neutrófilos activados provenientes de la circulación. Su degranulación libera citoquinas proinflamatorias (IL-17, IL-36), elastasas y granzima B, que aumentan la inflamación y conducen a la destrucción de la membrana basal y formación de ampollas subepidérmicas ${ }^{16,18}$. Por su parte, los queratinocitos locales producen enzimas que contribuyen a la formación de las vesículas/ampollas ${ }^{16}$.

\section{Clínica}

Las manifestaciones incluyen lesiones cutáneas, orales y gastrointestinales. El sello distintivo lo dan las manifestaciones cutáneas, consistentes en lesiones polimorfas, agrupadas, simétricas y distribuidas característicamente en superficies extensoras de codos, rodillas, región sacra y glúteos ${ }^{19}$ (Figura 3). Pero pueden aparecer también en la parte superior de la espalda, hombros, cuero ca- 


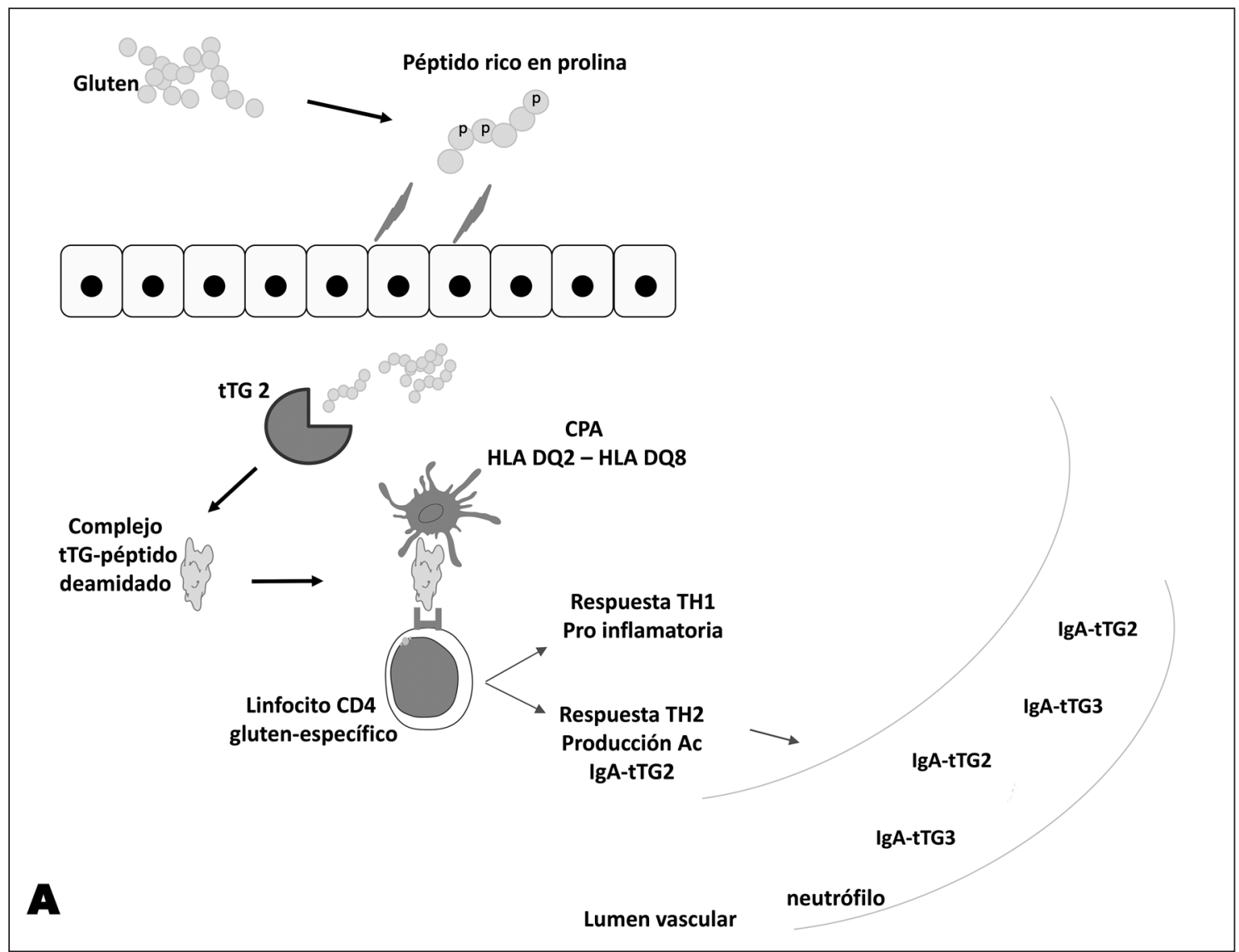

Figura 1. El gluten dietario es procesado por las enzimas digestivas, generando péptidos de gliadina que pasan a la lámina propria, donde interactúan con la transglutaminasa tisular (tTG2). Esta enzima los deamida, forma un complejo y la molécula queda cargada negativamente. El complejo deamidado es presentado por células presentadoras de antígeno a los linfocitos T CD4, a través de moléculas HLA-DQ2 o DQ8. La activación del linfocito T CD4 da lugar a la producción de citoquinas Th1 y metaloproteinasas de matriz, produciéndose daño celular epitelial y remodelación tisular de la mucosa intestinal. La respuesta de linfocitos B específicos para transglutaminasa 2 (tTG2) captan los complejos tTG2-gliadina y se estimula la producción de IgA anti-tTG2. Tanto los complejos antigénicos como los anticuerpos anti tTG2 salen a la circulación, donde también circula tTG3 o transglutaminasa epidérmica (eTG). Con el tiempo, se forma IgA anti-eTG debido a las similitudes estructurales de ambas transglutaminasas y todos circulan en el torrente sanguíneo.

belludo, nuca y cara ${ }^{11}$. Las lesiones elementales son pápulas eritematosas o vesículas agrupadas sobre una base eritematosa, a menudo reemplazadas por pápulas costrosas, excoriaciones y discromías debido al prurito intenso asociado. El prurito es el síntoma principal de DH y su ausencia es un fuerte argumento en contra del diagnóstico ${ }^{16}$. Se han reportado presentaciones atípicas, como lesiones purpúricas palmares o acrales, que hasta hoy no han sido reportadas en ningún otro trastorno ampolloso autoinmune ${ }^{20}$. En niños, los principales diagnósticos diferenciales son dermatitis atópica o numular, sarna, urticaria papular e impétigo. $\mathrm{Y}$ en adultos, el eccema recalcitrante, prurigo nodular, urticaria, penfigoide ampolloso y especialmente la dermatosis ampollosa IgA lineal, que se diferencia fundamentalmente porque al estudio de inmunofluorescencia directa (IFD) tiene un depósito de IgA lineal en la membrana basal ${ }^{19}$. Las manifestaciones orales consisten en erosiones de la mucosa oral y la lengua ${ }^{16}$. La sintomatología gastrointestinal es infrecuente y leve; sin embargo, 


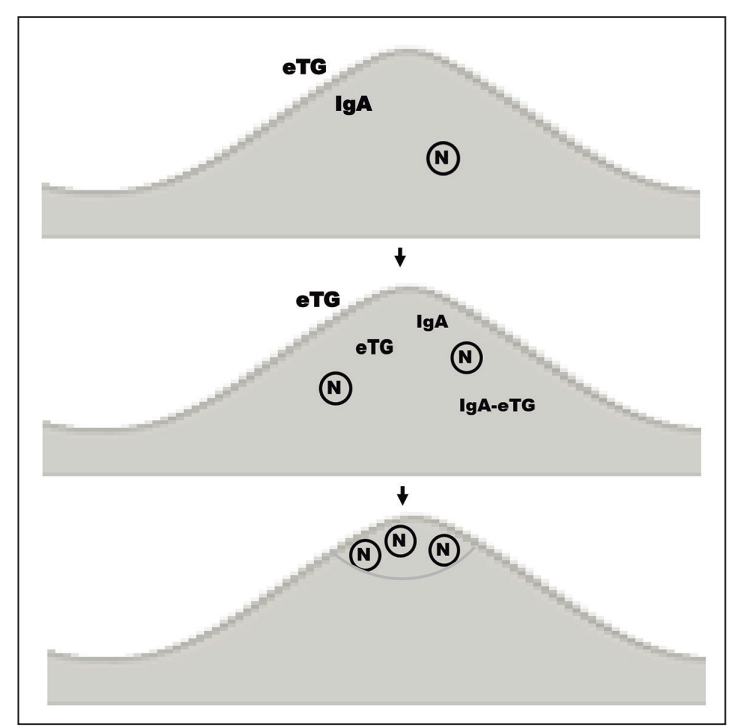

Figura 2. Cuando los anticuerpos IgA anti-eTG alcanzan la dermis, forman complejos con los antígenos (tTG3) producidos por los queratinocitos, que difunden hacia la dermis. Los complejos IgA-eTG en la dermis papilar provocan quimiotaxis de los neutrófilos (con formación de abscesos neutrófilos), ruptura de la lámina lúcida y formación de ampollas subepidérmicas.

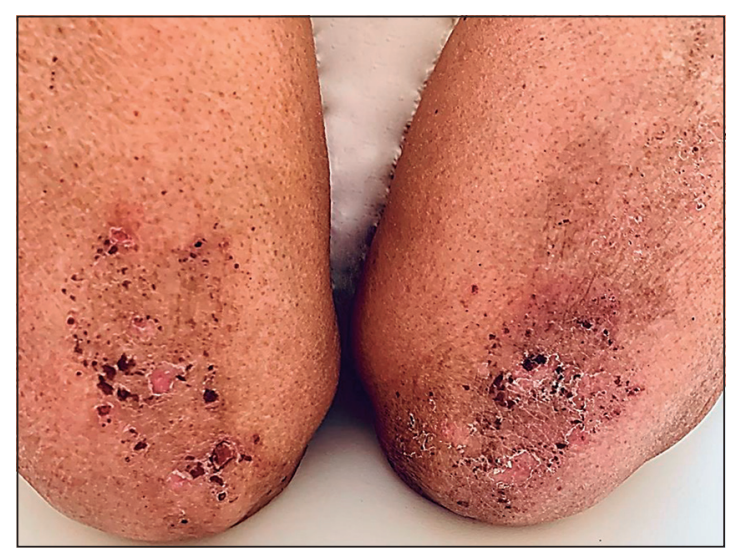

Figura 3. Lesiones típicas de dermatitis herpetiforme localizadas en las superficies extensoras como los codos, caracterizadas por lesiones polimorfas, agrupadas, simétricas, con escoriaciones y costras.

cerca de $70 \%$ de los pacientes con $\mathrm{DH}$ muestran el aplanamiento vellositario clásico de la $\mathrm{EC}^{22,23}$. Así, la mayoría de los pacientes con $\mathrm{DH}$ tienen hallazgos anormales en la biopsia intestinal, compatibles con una EC silente, definida como aquella que cursa con presencia de anticuerpos tTG2 positivos (AcTTG) y biopsia con lesión típica de la enfermedad, pero es asintomática.

Es interesante la observación que los pacientes celíacos con índice de masa corporal (IMC) $>25$ al momento del diagnóstico tienen más riesgo de tener otra enfermedad autoinmune, incluida la $\mathrm{DH}^{24}$. Por su parte, en $\mathrm{DH}$ se observó que el riesgo de desarrollar la enfermedad aumentó 14\% (IC del 95\%) por cada unidad de IMC; por el contrario, el riesgo de EC disminuyó 7\% (IC del 95\%) por unidad de $\mathrm{IMC}^{25}$.

\section{Trastornos asociados}

Existe un amplio espectro de patologías asociadas a EC/DH (Tabla 1). Debido a la malabsorción, los pacientes con $\mathrm{DH}$ tienen riesgo de anemia ferropriva, anemia megaloblástica, osteopenia y fracturas y trastornos tiroideos ${ }^{22,26}$. Al igual que en EC, el riesgo de linfoma no Hodgkin (LNH) y neoplasias malignas gastrointestinales está aumentado en DH. Sin embargo, en un gran estudio poblacional, la mortalidad solo aumentó durante los primeros 5 años del diagnóstico, sugiriendo que la DLG estricta protegería de esta complicación ${ }^{27}$.

\section{Diagnóstico}

El diagnóstico preciso de $\mathrm{DH}$ importa, porque el tratamiento requiere cambios considerables en la dieta, permanentes, y en la mayoría de los casos el uso de medicamentos potencialmente tóxicos. Los pilares diagnósticos se ilustran en la Tabla 2. El gold standard para el diagnóstico es la IFD ${ }^{19}$.

\section{Tabla 1. Principales condiciones descritas en asociación a enfermedad celíaca y dermatitis herpetiforme}

\begin{tabular}{|ll|}
\hline Tiroiditis autoinmune & Artritis reumatoide \\
\hline Diabetes mellitus tipo 1 & Dermatomiositis \\
\hline Anemia perniciosa & Polimiositis \\
\hline $\begin{array}{l}\text { Lupus eritematoso } \\
\text { sistémico }\end{array}$ & $\begin{array}{l}\text { Enfermedad inflamatoria } \\
\text { intestinal }\end{array}$ \\
Síndrome de Sjögren & Hepatitis autoinmune \\
Vitiligo & Psoriasis \\
Enfermedad de Addison & Vitíligo \\
\hline Nefropatía por IgA & Alopecia areata \\
\hline Déficit parcial de IgA & Ictiosis adquirida \\
\hline
\end{tabular}


Tabla 2. Pilares del diagnóstico de dermatitis herpetiforme

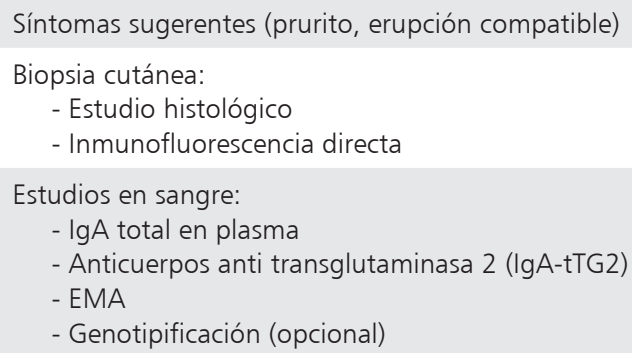

\section{Histopatología}

Depende de la presentación clínica y el tiempo de evolución. En los primeros dos días hay pápulas eritematosas y vesículas, se pueden evidenciar vesículas y ampollas subepidérmicas asociadas a acumulación de neutrófilos en las crestas papilares. En fases evolutivas tardías o en lesiones muy excoriadas, los hallazgos pueden ser inespecíficos. Los hallazgos histopatológicos por sí solos no permiten una diferenciación definitiva de otros trastornos ampollosos autoinmunes subepidérmicos, como la dermatosis ampollosa IgA lineal y penfigoide ampolloso ${ }^{20,28,29}$.

\section{Inmunofluorescencia directa}

La IFD detecta IgA en la piel no afectada; es una prueba simple, requiere una biopsia de piel perilesional y es la forma más confiable de establecer el diagnóstico. El hallazgo más frecuente es el depósito granular de IgA en las papilas dérmicas. Un patrón menos común es el granular continuo a lo largo de la unión dermoepidérmica, y ambos patrones pueden coexistir ${ }^{30}$ (Figura 4). Ocasionalmente, y hasta en $50 \%$ de los pacientes japoneses, se puede encontrar depósito fibrilar de IgA ${ }^{12}$. Los falsos negativos pueden observarse hasta en 10\% de los casos de DH y se describen en tres circunstancias, cuando la biopsia (i) se obtiene de piel afectada o (ii) en un área nunca involucrada o (iii) si el paciente ha estado en DLG por muchos años $^{22}$. Un estudio reciente en pacientes celíacos con enfermedades cutáneas inflamatorias no DH encontró presencia de depósitos granulares de IgA en piel sana perilesional en todos los pacientes ${ }^{31}$, lo que sugiere que estos depósitos podrían ser un marcador inmunopatológico de EC. Estudios previos habían reportado estos hallazgos en piel sana de pacientes celíacos sin manifestaciones cutáneas, además de colocalización IgA/tTG3 en los depósitos granulares ${ }^{32}$. Ambos estudios enfatizan la importancia de la correlación clínico-patológica.

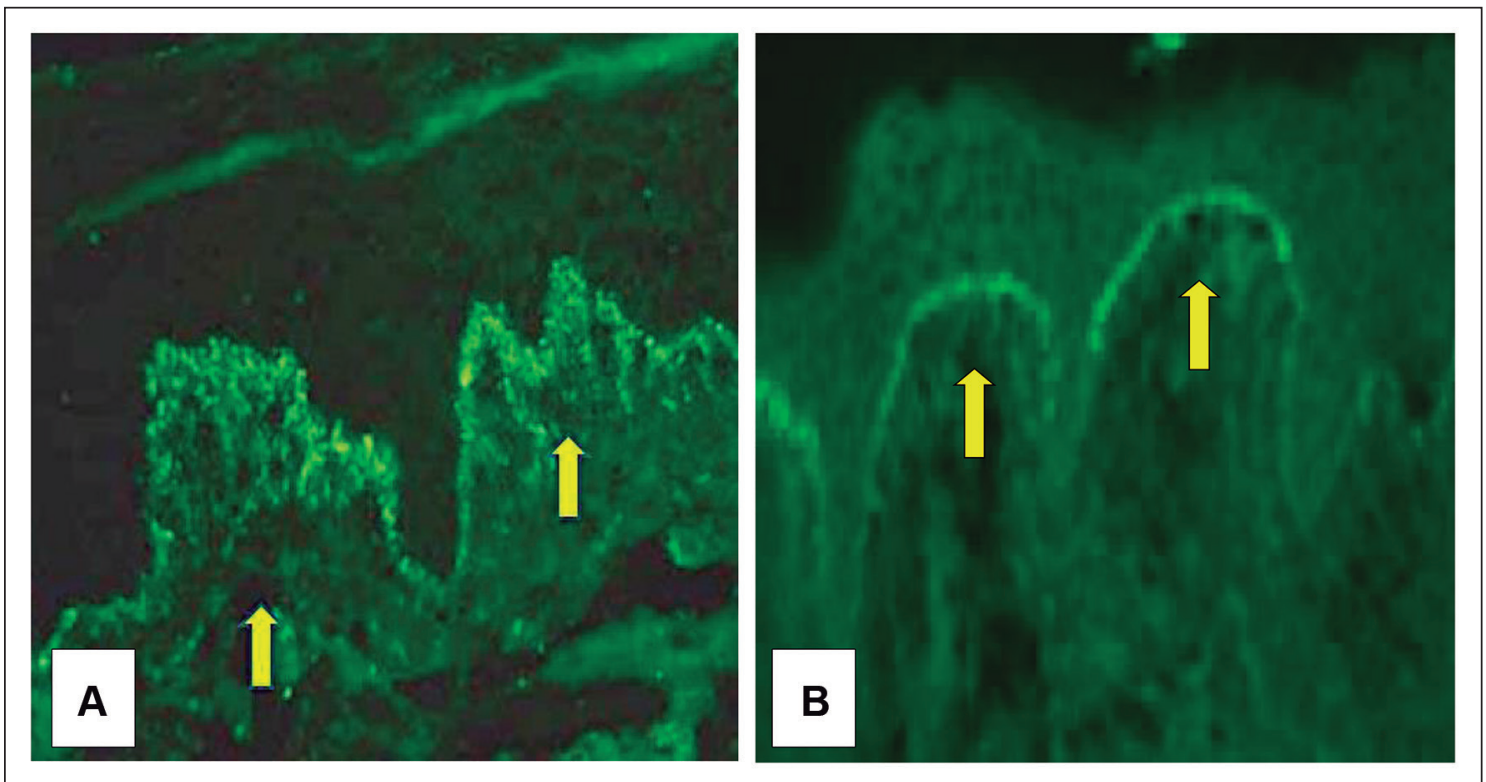

Figura 4. Inmunofluorescencia ilustrando la presencia de IgA en piel no afectadacomo depósito granular de lgA en las papilas dérmicas (panel A) y depósito granular continuo a lo largo de la unión dermoepidérmica (panel B). 
Por lo tanto, los pacientes con EC que presenten manifestaciones cutáneas con IFD positiva deben ser investigados para hacer el diagnóstico diferencial entre DH y otras enfermedades inflamatorias de la piel.

\section{Serología}

Los AcTTG y antiendomisio (EMA) han demostrado ser altamente precisos para diagnóstico de $\mathrm{EC}^{3}$. En $\mathrm{DH}$, su presencia junto al cuadro clínico compatible sugieren el diagnóstico, pero tienen baja sensibilidad. En un estudio finlandés, el 11\% de los pacientes DH con atrofia vellositaria grave y el $30 \%$ con atrofia vellositaria parcial tenían niveles normales de AcTTG, lo que indica que un resultado negativo no excluye $\mathrm{DH}^{23}$. Aunque actualmente estos anticuerpos están cuestionados para hacer seguimiento de EC, en la práctica clínica se utilizan para evaluar adherencia a $\mathrm{DLG}^{33}$. No son útiles para hacer seguimiento de las lesiones dérmicas. Los anticuerpos anti-PGD no están validados para el estudio diagnóstico de $\mathrm{DH}$.

\section{IgA total}

Hasta la fecha no se ha descrito déficit selectivo de IgA en DH, pero sí déficit parcial, lo que suele retrasar el diagnóstico ${ }^{34}$. Por esto, se considera necesario medir las concentraciones de $\operatorname{IgA}$ al mismo tiempo que los AcTTG o EMA.

\section{Otras pruebas diagnósticas}

Aunque la presencia de HLA-DQ2/DQ8 es alta entre los pacientes $\mathrm{DH}^{7}$, la especificidad de estos alelos es pobre, por lo que actualmente su estudio no se incluye en el protocolo inicial de diagnóstico de $\mathrm{EC} / \mathrm{DH}^{2,3,19}$. Por su alto valor predictivo negativo, se reservan para excluir el diagnóstico de $\mathrm{DH}$ y evitar exámenes innecesarios ${ }^{16}$. En Latinoamérica (México, Brasil, Argentina) ${ }^{14}$ hasta $10 \%$ de los pacientes EC pueden no portar estos genes, por lo que su rol en el proceso diagnóstico de EC en la región está en discusión. No se encontró información de este tipo en DH. La biopsia de intestino delgado es mandatoria para el diagnóstico de $\mathrm{EC}^{3}$, pero no todos los autores la consideran indispensable para el diagnóstico de $\mathrm{DH}^{19,33}$. La evidencia reciente indica que en pacientes con $\mathrm{DH}$, con o sin atrofia vellositaria, no hay diferencias en la respuesta a la DLG, calidad de vida, presencia de enfermedades crónicas o complicaciones asociadas a $\mathrm{EC}^{27,33}$, lo que respalda la política actual de no obtener biopsias de intestino delgado rutinarias en pacientes con $\mathrm{DH}$.

Considerando la alta incidencia de condiciones asociadas, el estudio inicial de pacientes con DH debe incluir medición de glucosa en ayunas, pruebas tiroideas, anticuerpos antiperoxidasa tiroidea, anticuerpos anticélulas parietales gástricas, anticuerpos antinucleares y anti-Ro ${ }^{19}$. La búsqueda de deficiencias nutricionales debe incluir medición de nutrición de hierro, ácido fólico, vitamina $\mathrm{D}$ y vitamina $\mathrm{B} 12^{3}$. Además, se sugiere el cribado en los familiares de primer grado ${ }^{3,19}$.

\section{Tratamiento}

El objetivo inicial es la resolución del prurito y lesiones cutáneas mediante el tratamiento farmacológico. El control a largo plazo requiere DLG estricta permanente, ya que tanto la enteropatía como la erupción cutánea dependen del gluten. El manejo debe estar a cargo de especialistas, dermatólogo y gastroenterólogo, y una nutricionista especializada que supervise la DLG.

\section{$D L G$}

Es el tratamiento de elección, independiente de si hay o no atrofia vellositaria intestinal ${ }^{35}$. La evidencia de la eficacia de la DLG en el tratamiento de las manifestaciones cutáneas y gastrointestinales de DH es contundente, sin embargo, es conocido que la resolución de los síntomas cutáneos tarda más (aproximadamente 2 años) que la respuesta intestinal (3-6 meses), y que las lesiones cutáneas recurren invariablemente dentro de 12 semanas posterior a la reintroducción de gluten ${ }^{19}$. La DLG excluye el consumo de trigo, cebada, centeno y avena. Hasta hoy se discute la seguridad del consumo de avena en EC, pero existe poca evidencia en $\mathrm{DH}$. Un estudio reciente demostró que el consumo de avena no contaminada es seguro entre pacientes finlandeses con $\mathrm{DH}$, siendo su calidad de vida mejor que la de aquellos que no consumían avena $^{36}$. Por otro lado, la capacidad de provocar inflamación de EC mediados por tTG2 varía significativamente entre las variedades de avena ${ }^{37}$.

La ingesta voluntaria o involuntaria de gluten puede revelarse por el aumento de $\mathrm{AcTTG}^{33}$, pero no hay biomarcadores específicos para evaluar adecuadamente el grado de inflamación de la mu- 
cosa, que puede ocurrir con cantidades variables de gluten.

La EC refractaria es una condición en la que no hay respuesta clínica o histológica a la DLG estricta, es infrecuente $(1,7 \%)$ y se caracteriza por manifestaciones cutáneas que persisten a pesar de DLG estricta a largo plazo. En pacientes DH se ha reportado que la erupción cutánea persistió a pesar de la DLG y necesitaron el uso de dapsona, pese a que mantenían DLG estricta durante una media de 16 años; la mayoría mantuvo depósitos de IgA en la piel, pero a diferencia de la EC refractaria, no presentaron alteraciones en la biopsia intestinal, ni complicaciones como malabsorción o linfoma, lo que se interpretó como que la condición de refractariedad en $\mathrm{DH}$ difiere de la $\mathrm{EC}^{38}$.

\section{Dapsona}

La dapsona es un bacteriostático que inhibe la quimiotaxis y reduce el daño tisular mediado por neutrófilos en los sitios lesionales ${ }^{39}$. Es el fármaco de elección para la supresión de los síntomas de la DH. Se prescribe 6 a 24 meses, hasta que la DLG sea eficaz ${ }^{40}$, ya que la dapsona solo controla la erupción pero no tiene efecto sobre la enteropatía ${ }^{35}$. Su efecto sobre la dermatosis es dramático, alivia el prurito dentro de 48-72 horas, resuelve las lesiones cutáneas en días, pero las lesiones recurren 24-48 horas posinterrupción del tratamiento $^{39}$. Se inicia con una dosis baja (25-50 mg/ día) para minimizar los efectos secundarios y, de ser necesario, puede aumentarse gradualmente hasta $100-200 \mathrm{mg} /$ día $^{35,40}$. Los efectos adversos dependen de la dosis y son más frecuentes en pacientes con comorbilidades (anemia, enfermedad cardiopulmonar y hepatopatías graves), deficiencia de glucosa-6-fosfato deshidrogenasa (G6PD) y comedicación con otros inductores de metahemoglobinemia (sulfonamidas y anestésicos locales) ${ }^{41}$; se clasifican en tóxicos, incluyendo anemia hemolítica y metahemoglobinemia, e idiosincrásicos ${ }^{19,39}$. Entre estos últimos, el síndrome de hipersensibilidad a la dapsona se considera el más grave; ocurre dentro de 3 a 20 semanas de tratamiento en $\sim 1,4 \%$ de los pacientes. Se manifiesta con fiebre, fotosensibilidad, exantema, síntomas gastrointestinales y compromiso hepático, que puede llegar a la insuficiencia hepática. Otras características clínicas frecuentes incluyen linfadenopatías, leucocitosis, anemia y eosinofilia ${ }^{19,41}$. Debido a estos riesgos, antes de iniciar la terapia con dapsona, en los pacientes debe obtenerse una historia clínica y examen físico completo, y solicitar hemograma, metahemoglobina, recuento de reticulocitos, función renal, función hepática, serología de hepatitis y G6PD ${ }^{41}$. El efecto secundario más común de la dapsona es la hemólisis, y los pacientes deben ser controlados dentro de las 2 primeras semanas de inicio del medicamento ${ }^{19,41}$.

\section{Fármacos alternativos}

La sulfasalazina y la sulfametoxipiridazina se usan como alternativa eficaz de la dapsona. Las dosis sugeridas son de 1-2 g/día para la sulfasalazina y $0,25-1,5 \mathrm{~g} /$ día para la sulfametoxipiridazina. En ambas, los efectos adversos más comunes son gastrointestinales (náuseas, vómitos, anorexia). La anemia hemolítica, las reacciones de hipersensibilidad, proteinuria y cristaluria son menos comunes. Los pacientes deben ser monitoreados con hemograma completo, creatinina y análisis de orina antes del tratamiento, mensualmente en los primeros 3 meses y cada 6 meses posteriormente ${ }^{19}$. En casos refractarios o con contraindicación a los fármacos tradicionales, se han descrito casos clínicos tratados con inmunosupresores convencionales, como azatioprina, mofetil micofenolato y ciclosporina. En un paciente con DH refractaria se logró remisión clínica y serológica completa utilizando rituximab como coadyuvante de azatioprina $^{42}$.

\section{Seguimiento}

Los controles clínicos y el monitoreo con exámenes de laboratorio deben ser periódicos e indefinidos, más frecuentes durante los primeros 6 meses de tratamiento, en especial si se administra dapsona ${ }^{26}$ (Tabla 2). Los objetivos son educar al paciente, indicar el cribado de familiares de primer grado, monitorear la adherencia a la DLG y la respuesta de la sintomatología, detectar comorbilidades o complicaciones. El tiempo para normalizar los AcTTG depende del nivel inicial, habitualmente se consigue después de dos años de $\mathrm{DLG}^{43}$.

\section{Conclusión}

La DH es una enfermedad ampollar autoinmune crónica, caracterizada por la presencia de lesiones pápulo-vesiculosas, intensamente pruri- 
ginosas, de distribución simétrica en las superficies de extensión de miembros y región sacra. Aunque es una manifestación de EC, la enteropatía inducida por gluten es habitualmente asintomática. De esta manera, el compromiso cutáneo es la puerta de entrada para la detección de la afectación intestinal, su tratamiento y prevención de complicaciones a largo plazo.

\section{Referencias}

1. Encuesta Nacional de Salud, CHILE, 2009-2010. (Último acceso: 18 de abril de 2020). http://ghdx.healthdata. org/record/chile-national-health-survey-2009-2010.

2. Husby S, Koletzko S, Korponay-Szabó IR, Mearin ML, Phillips A, Shamir R, et al. European Society for Pediatric Gastroenterology, Hepatology, and Nutrition guidelines for the diagnosis of coeliac disease. J Pediatr Gastroenterol Nutr. 2012; 54 (1): 136-60. doi: 10.1097/ MPG.0b013e31821a23d0.

3. Rubio-Tapia A, Hill ID, Kelly CP, Calderwood AH, Murray JA; American College of Gastroenterology. ACG clinical guidelines: diagnosis and management of celiac disease. Am J Gastroenterol. 2013; 108 (5): 656-76. doi: 10.1038/ajg.2013.79.

4. Salmi T, Hervonen K, Kautiainen H, Collin P, Reunala T. Prevalence and incidence of dermatitis herpetiformis: a 40 - year prospective study from Finland. Br J Dermatol. 2011; 165 (2): 354-9. doi: 10.1111/j.13652133.2011.10385.x.

5. West J, Fleming KM, Tata LJ, Card TR, Crooks CJ. Incidence and prevalence of celiac disease and dermatitis herpetiformis in the UK over two decades: population-based study. Am J Gastroenterol. 2014; 109 (5): 757-68. doi: 10.1038/ajg.2014.55.

6. Marks J, Shuster S, Watson A.J. Small-bowel changes in dermatitis herpetiformis. Lancet. 1966; 2 (7476): 1280-2. doi: 10.1016/s0140-6736(66)91692-8.

7. Spurkland A, Ingvarsson G, Falk ES, Knutsen I, Sollid LM, Thorsby E. Dermatitis herpetiformis and celiac disease are both primarily associated with the HLA-DQ (alpha $1^{\star} 0501$, beta $1^{\star} 02$ ) or the HLA-DQ (alpha $1^{\star} 03$, beta $1^{\star} 0302$ ) heterodimers. Tissue Antigens. 1997; 49 (1): 29-34. doi: 10.1111/j.1399-0039.1997.tb02706.x.

8. Dieterich W, Ehnis T, Bauer M, Donner P, Volta U, Riecken EO, et al. Identification of tissue transglutaminase as the autoantigen of celiac disease. Nat Med. 1997; 3 (7): 797-801. doi: 10.1038/nm0797-797.

9. Dieterich W, Laag E, Bruckner-Tuderman L, Reunala T, Kárpáti S, Zágoni T, et al. Antibodies to tissue transglu- taminase as serologic markers in patients with dermatitis herpetiformis. J Invest Dermatol. 1999; 113 (1): 133-6. doi: 10.1046/j.1523-1747.1999.00627.x.

10. Sárdy M1, Kárpáti S, Merkl B, Paulsson M, Smyth N. Epidermal transglutaminase (TGase 3) is the autoantigen of dermatitis herpetiformis. J Exp Med. 2002; 195 (6): 747-57. doi: 10.1084/jem.20011299.

11. Bolotin D, Petronic-Rosic V. Dermatitis herpetiformis. Part I. Epidemiology, pathogenesis, and clinical presentation. J Am Acad Dermatol. 2011; 64 (6): 1017-24. doi: 10.1016/j.jaad.2010.09.777.

12. Ohata C, Ishii N, Niizeki H, Shimomura Y, Furumura $\mathrm{M}$, Inoko $\mathrm{H}$, et al. Unique characteristics in Japanese dermatitis herpetiformis. Br J Dermatol. 2016; 174 (1): 180-3. doi: 10.1111/bjd.13965.

13. Lebwohl B, Sanders DS, Green PHR. Coeliac disease. Lancet. 2018; 391 (10115): 70-81. doi: 10.1016/ S0140-6736(17)31796-8.

14. Araya M, Oyarzún A, Lucero Y, Espinosa N, Pérez-Bravo F. DQ2, DQ7 and DQ8 Distribution and Clinical Manifestations in Celiac Cases and Their First-Degree Relatives. Nutrients. 2015;7(6):4955-65. doi: 10.3390/ nu7064955.

15. Clarindo MV, Possebon AT, Soligo EM, Uyeda H, Ruaro RT, Empinotti JC. Dermatitis herpetiformis: pathophysiology, clinical presentation, diagnosis and treatment. An Bras Dermatol. 2014; 89 (6): 865-77. doi: 10.1590/ abd1806-4841.20142966.

16. Antiga E, Maglie R, Quintarelli L, Verdelli A, Bonciani D, Bonciolini V, et al. Dermatitis Herpetiformis: Novel Perspectives. Front Immunol. 2019; 10:1290. doi: 10.3389/fimmu.2019.01290.

17. Hietikko M, Hervonen K, Ilus T, Salmi T, Huhtala H, Laurila K, et al. Ex vivo Culture of Duodenal Biopsies from Patients with Dermatitis Herpetiformis Indicates that Transglutaminase 3 Antibody Production Occurs in the Gut. Acta Derm Venereol. 2018; 98 (3): 366-372. doi: 10.2340/00015555-2849.

18. Zebrowska A, Wozniacka A, Juczynska K, Ociepa K, Waszczykowska E, Szymczak I, et al. Correlation between IL36alpha and IL17 and activity of the disease in selected autoimmune blistering diseases. Mediators Inflamm. 2017; 2017: 8980534. doi: 10.1155/2017/8980534.

19. Caproni M, Antiga E, Melani L, Fabbri P. Italian Group for Cutaneous Immunopathology. Guidelines for the diagnosis and treatment of dermatitis herpetiformis. J Eur Acad Dermatol Venereol. 2009; 23 (6): 633-8. doi: 10.1111/j.1468-3083.2009.03188.x.

20. Rose C, Bröcker EB, Zillikens D. Clinical, histological and immunpathological findings in 32 patients with dermatitis herpetiformis Duhring. J Dtsch Dermatol 
Ges. 2010; 8 (4): 265-70, 265-71. doi: 10.1111/j.16100387.2009.07292.x.

21. Lähteenoja H, Irjala K, Viander M, Vainio E, Toivanen A, Syrjänen S. Oral mucosa is frequently affected in patients with dermatitis herpetiformis. Arch Dermatol. 1998; 134 (6): 756-8. doi: 10.1001/archderm.134.6.756.

22. Alonso-Llamazares J, Gibson LE, Rogers RS 3rd. Clinical, pathologic, and immunopathologic features of dermatitis herpetiformis: review of the Mayo Clinic experience. Int J Dermatol. 2007; 46 (9): 910-9. doi: 10.1111/j.1365-4632.2007.03214.x.

23. Mansikka E, Hervonen K, Salmi TT, Kautiainen H, Kaukinen K, Collin P, Reunala T. The Decreasing Prevalence of Severe Villous Atrophy in Dermatitis Herpetiformis: A 45-Year Experience in 393 Patients. J Clin Gastroenterol. 2017; 51(3) :235-9. doi: 10.1097/ MCG.0000000000000533.

24. Conti L, Lahner E, Galli G, Esposito G, Carabotti M, Annibale B. Risk Factors Associated with the Occurrence of Autoimmune Diseases in Adult Coeliac Patients. Gastroenterol Res Pract. 2018; 2018: 3049286. Doi: 10.1155/2018/3049286.

25. Harpsøe MC, Basit S, Andersson M, Nielsen N, Frisch $\mathrm{M}$, Wohlfahrt J, et al. Body mass index and risk of autoimmune diseases: a study within the Danish National Birth Cohort. Int J Epidemiol. 2014; 43 (3): 843-55. doi: 10.1093/ije/dyu045.

26. Vale ECSD, Dimatos OC, Porro AM, Santi CG. Consensus on the treatment of autoimmune bullous dermatoses: dermatitis herpetiformis and linear IgA bullous dermatosis - Brazilian Society of Dermatology. An Bras Dermatol. 2019; 94 (2 Suppl 1): 48-55. doi: 10.1590/ abd1806-4841.2019940208.

27. Hervonen K, Alakoski A, Salmi TT, Helakorpi S, Kautiainen $\mathrm{H}$, Kaukinen $\mathrm{K}$, et al. Reduced mortality in dermatitis herpetiformis: a population-based study of 476 patients. Br J Dermatol. 2012; 167 (6): 1331-1337. doi: 10.1111/j.1365-2133.2012.11105.x.

28. Fuertes I, Mascaró JM, Bombí JA, Iranzo P. Estudio retrospectivo de las características clínicas, histológicas e inmunológicas de los pacientes con dermatitis herpetiforme. Experiencia del Hospital Clínic de Barcelona entre los años 1995 y 2010 y revisión de la literatura. Actas Dermosifiliogr. 2011; 102 (9): 699-705. doi: 10.1016/j. ad.2010.11.007.

29. Warren SJ, Cockerell CJ. Characterization of a subgroup of patients with dermatitis herpetiformis with nonclassical histologic features. Am J Dermatopatholy. 2002; 24 (4): 305-8. doi: 10.1097/00000372-200208000-00003.

30. Barnadas MA. Dermatitis Herpetiformis: A Review of Direct Immunofluorescence Findings. Am
J Dermatopathol. 2016; 38 (4): 283-8. doi: 10.1097/ DAD.0000000000000420.

31. Bonciolini V, Antiga E, Bianchi B, Del Bianco E, Ninci A, Maio V, et al. Granular IgA deposits in the skin of patients with coeliac disease: is it always dermatitis herpetiformis? Acta Derm Venereol. 2019; 99 (1): 78-83. doi: 10.2340/00015555-3001.

32. Cannistraci C, Lesnoni La Parola I, Cardinali G, Bolasco G, Aspite N, Stigliano V, et al. Co-localization of IgA and TG3 on healthy skin of coeliac patients. J Eur Acad Dermatol Venereol. 2007; 21 (4): 509-14. doi: 10.1111/j.1468-3083.2006.02017.x.

33. Salmi T, Hervonen K. Current Concepts of Dermatitis Herpetiformis. Acta Derm Venereol. 2020; 100 (5): adv00056. doi: 10.2340/00015555-3401.

34. Samolitis NJ, Hull CM, Leiferman KM, Zone JJ. Dermatitis herpetiformis and partial IgA deficiency. J Am Acad Dermatol. 2006; 54 (5 Suppl): S206-9. doi: 10.1016/j. jaad.2005.06.033.

35. Garioch JJ, Lewis HM, Sargent SA, Leonard JN, Fry L. 25 years' experience of a gluten-free diet in the treatment of dermatitis herpetiformis. Br J Dermatol. 1994; 131 (4): 541-5. doi: 10.1111/j.1365-2133.1994.tb08557.x.

36. Alakoski A, Hervonen K, Mansikka E, Reunala T, Kaukinen K, Kivelä L, et al. The Long-Term Safety and Quality of Life Effects of Oats in Dermatitis Herpetiformis. Nutrients. 2020; 12 (4):1060. doi: 10.3390/nu12041060.

37. Silano M, Pozo EP, Uberti F, Manferdelli S, Del Pinto $\mathrm{T}$, Felli C, et al. Diversity of oat varieties in eliciting the early inflammatory events in celiac disease. Eur J Nutr. 2014; 53 (5): 1177-86. doi: 10.1007/s00394-013-0617-4.

38. Hervonen K, Salmi TT, Ilus T, Paasikivi K, Vornanen $\mathrm{M}$, Laurila $\mathrm{K}$, et al. Dermatitis herpetiformis refractory to gluten-free dietary treatment. Acta Derm Venereol. 2016; 96 (1): 82-6. doi: 10.2340/00015555-2184.

39. Coleman MD. Dapsone: modes of action, toxicity and possible strategies for increasing patient tolerance. $\mathrm{Br} \mathrm{J}$ Dermatol. 1993; 129 (5):507-13. doi: 10.1111/j.13652133.1993.tb00476.x

40. Ghaoui N, Hanna E, Abbas O, Kibbi AG, Kurban M. Update on the use of dapsone in dermatology. Int J Dermatol. 2020; 59 (7): 787-95. doi: 10.1111/ijd.14761.

41. Wozel G, Blasum C. Dapsone in dermatology and beyond. Arch Dermatol Res. 2014; 306 (2): 103-124. doi: 10.1007/s00403-013-1409-7.

42. Albers LN, Zone JJ, Stoff BK, Feldman RJ. Rituximab Treatment for Recalcitrant Dermatitis Herpetiformis. JAMA Dermatol. 2017; 153 (3): 315-8. doi: 10.1001/ jamadermatol.2016.4676.

43. Fry L. Dermatitis herpetiformis: problems progress and prospects. Eur J Dermatol 2002; 12 (6): 523-31. 